TITLE:

\title{
Pigeons (Columba livia) integrate visual motion using the vector average rule: effect of viewing distance
}

\section{AUTHOR(S):}

Hataji, Yuya; Fujita, Kazuo; Kuroshima, Hika

\section{CITATION:}

Hataji, Yuya ... [et al]. Pigeons (Columba livia) integrate visual motion using the vector average rule: effect of viewing distance. Animal Cognition 2020, 23: 819-825

\section{ISSUE DATE:}

2020

URL:

http://hdl.handle.net/2433/253558

\section{RIGHT:}

This is a post-peer-review, pre-copyedit version of an article published in 'Animal cognition'. The final authenticated version is available online at: https://doi.org/10.1007/s10071-020-01376-1.; The full-text file will be made open to the public on 02 April 2021 in accordance with publisher's 'Terms and Conditions for Self-Archiving; この論文は出版社版て ありません。引用の際には出版社版をご確認じ利用ください。;This is not the published version. Please cite only the published version. 
1 Pigeons (Columba livia) integrate visual motion using the vector

2 average rule: Effect of viewing distance

3 Yuya Hataji

4 Kazuo Fujita

$5 \quad$ Hika Kuroshima

6

7 Affiliation: Kyoto University

8 Affiliation address: Department of Psychology, Graduate School of Letters, Kyoto

9 University, Yoshida-honmachi, Sakyo, Kyoto 606-8501, Japan.

10 Corresponding author: Yuya Hataji, yuya.hataji@gmail.com

11 Telephone/Fax: +81757532442

12 


\section{Abstract}

14 Integrating local motion signals detected by the primary motion detector is crucial

15 for representing a rigid, two-dimensional motion. The nature of motion integration

16 has been studied using stimuli consisting of two superimposed sinusoidal gratings

17 of different orientations, called plaid motion, and it has been shown that humans

18 perceive integrated motion in the direction where the component constraint lines

19 are intersected. We previously found that pigeons and humans perceive different

20 movement directions from plaid motion; pigeons responded to the vector average

21 direction of the gratings. Although this suggests that the underlying processes of

motion integration differ between the two species, the viewing distance in the

23 pigeon experiment, which used a touch panel procedure, was much smaller than in

24 typical human experiments. The current study investigated the potential effect of

viewing distance on perception of plaid motion in pigeons. We trained six pigeons to

detect whether motion directions were tilted leftward or rightward while a visual

display was presented 0 or $40 \mathrm{~cm}$ from an operant chamber. The pigeons responded

to plaid stimuli for both viewing distance conditions as if they perceived motion in 
31 suggests that pigeons use a different strategy than humans for integrating visual

32 motion.

33 Keywords: visual motion, plaid motion, viewing distance, pigeons 


\section{Introduction}

35 Motion processing is important for visually capable animals as it provides

36 information about prey, predators and conspecifics as well as information about

37 self-motion through optical flow signals. The most basic stage of visual motion

38 processing detects a spatiotemporal change in visual cues (primarily luminance)

39 within local receptive fields (Adelson and Bergen 1985). These 1D motion signals

40 with different preferred locations in the visual field and preferred orientations are

41 then integrated in higher processing stages to represent a rigid $2 \mathrm{D}$ motion

42 (Movshon and Newsome 1996). The rule of 1D motion integration across

43 orientations has been explored using plaid motion stimuli (Figure 1B, C, see also

44 Supplementary Movie S1, S2), in which two gratings drifting in different directions

45 are superimposed (Adelson and Movshon 1982). Due to the aperture problem

46 inherent in the 1D motion detector, 1D motion signals from component gratings are

47 ambiguous in speed and directions; there is a family of possible vectors that lie on

48 the constraint line along the orientation of the grating.

49 Humans perceive integrated 2D motion of plaid in the direction consistent with the

50 intersection of constraint lines (IOC) of components (blue dotted arrows in Figure

51 1B, C) (Adelson and Movshon 1982). By contrast, at least one avian species, 
pigeons (Columba livia), does not appear to integrate 1D motion components based

stimulus surrounded by numerous pecking keys on a touch monitor, and trained to

stimulus was presented, the pigeons did not respond based on the IOC rule, but

pecked the keys positioned at the vector average (VA) of the two components (red

solid arrows in Figure 1B, C). This result implies that motion integration strategies

differ across vertebrate species. This could be due on a proximal level to their neural architectures (Shimizu and Watanabe 2012), ultimately driven by varying ecological requirements (e.g. flying control, Wylie et al. 2018).

Behavioral studies of perceptual characteristics in non-human animals (hereafter:

"animals"), require consideration of whether the data do reflect the nature of perceptual processing of that animal or the variability of testing methods used across species. In particular, many studies of comparative vision require animals to touch visual stimuli with their nose or beak (for review, Qadri and Cook 2015).

67 Compared to typical psychophysical experiments with humans, this touch panel method results in a large visual angle of the stimulus with the stimulus size enlarged in the visual field; it possibly also induces attentional allocations to local 
70 features. For example, pigeons and bantams (Gallus gallus domesticus) perceive the Ebbinghaus illusion in the opposite direction to typical humans (Nakamura et al. 2008, 2014). In humans, the Ebbinghaus illusion was modulated when distant portions of the inducer are erased (Weintraub 1979). Therefore, the birds possibly ignore peripheral parts of the inducer due to their closer viewing distances (but see study showed that a visual size illusion was abolished in bumble bees when the viewing distance was restricted (Howard et al. 2017).

Similar to the Ebbinghaus illusion, humans perceive a plaid motion stimulus in different directions according to the viewing conditions (Yo and Wilson 1992). Although we typically perceive a plaid stimulus moving in the IOC direction, we perceive the VA direction when the stimulus is presented briefly or in a peripheral visual field. Given that humans use VA and IOC solutions to motion integration according to viewing conditions (Bowns and Alais 2006), it is possible that the species difference in motion integration reported by Hataji et al. (2019) was attributable to the difference in viewing distance. Therefore, the present study tested whether the perception of plaid motion in pigeons is affected by viewing 
88 stimulus tilted left- or rightward (Goto \& Lea, 2003). For training stimuli, we used

89 random dot motion and type 1 plaid motion (where the two component gratings of

90 the plaid move such that the VA and IOC directions are the same) (Figure 1A, B).

91 After the pigeons leaned the discrimination task, we presented the type 2 plaid, in

92 which VA and IOC directions were dissociated (Figure 1C). The training and

93 testing were done using 0 and $40 \mathrm{~cm}$ viewing distances. If pigeons responded to a

94 type 2 plaid stimulus in the same manner in both viewing conditions, this would

95 strongly suggest that pigeons integrate visual motion differently than humans.

96

$97 \quad$ Methods

$98 \quad$ Subjects

99 Six male pigeons participated (mean age: 4.5 years, range: 2-9). They were

100 individually housed and maintained at $80-90 \%$ of their free-feeding weights. They

101 had participated in several visual discrimination studies, and three had

102 participated in a motion discrimination study (S2, S3 and S6, Hataji et al. 2019).

103 Water and grit were freely available in the home cage. The experiment adhered to

104 the ethical guidelines of Kyoto University, and was approved by the Animal

105 Experiments Committee of the Graduate School of Letters, Kyoto University (No. 
The experiments were conducted in an operant chamber (W/D/H was 41/31/40 cm)

110 (Figure 2A, B). A transparent plexiglass occluded the window through which the

111 subjects viewed the visual stimulus on a 15 in. LCD monitor (EIZO, FlexScan

112 L367). The distance between the window and monitor was 0 or $40 \mathrm{~cm}$, depending

113 on the experimental phase. The distance of $40 \mathrm{~cm}$ was chosen because pigeons can

114 reliably discriminate the movement direction of grating patterns at that distance

115 (Martinoya and Bloch 1983). The visual angles of the stimulus (119 mm diameter)

116 were 70 and $14^{\circ}$ for 0 and $40 \mathrm{~cm}$ viewing conditions, respectively, assuming a

117 distance of $85 \mathrm{~mm}$ between the bird's head and the window, as obtained from a

118 head tracking study (unpublished data). The space including the chamber window

119 and the monitor was covered with black corrugated plastic boards, preventing the

120 subject from seeing the room in the $40 \mathrm{~cm}$ condition. Three response keys with

different LED light colors were attached at the top, left and right of the window. A grain hopper delivered food rewards through an opening on the left-side wall. The experiments were controlled by a personal computer (ThirdWave Corporation, 
124 Diginnos Series) and a digital input/output module (Contec, DIO-16/16(USB)),

125 running MATLAB with the Psychtoolbox extensions (Brainard 1997).

126 Stimuli

127 Random dot motion and type 1 plaid motion were used as training stimuli (Figure

128 1A, B). The stimulus window was created based on the 2D Gaussian function

129 flattened by the cumulative Gaussian function. The window started to fade around

$13070.1 \mathrm{~mm}$-diameter and alpha value was below 0.1 around $119 \mathrm{~mm}$-diameter. In the

131 stimulus window, white and black dots moved in the same direction on a gray

132 background. The number, size and speed of dots were randomly determined in

133 every trial to prevent discrimination based on potential local features (number: 30

$134-50$, size: $8.9-14.9 \mathrm{~mm}$, speed: $35.6-142.6 \mathrm{~mm} / \mathrm{s})$. The type 1 plaid stimulus was

135 composed of two sinusoidal gratings of different orientations. The spatial

136 frequencies and speeds of both component gratings were the same within a trial,

137 which resulted in the VA and IOC directions being the same. The spatial frequency,

138 speed and relative orientation of the plaid were randomly determined in every trial

139 to prevent discrimination based on potential local features (spatial frequency: 11.9

$140-29.7 \mathrm{~mm} /$ cycle, speed: $35.6-142.6 \mathrm{~mm} / \mathrm{s}$, relative orientation: $\left.15-90^{\circ}\right)$. The

141 direction of dot motion and integrated direction of type 1 plaid was either $\pm 18,54$, 
14290,126 or $162^{\circ}$ from upward direction.

143 Type 2 plaid motion was used as the probe stimulus (Figure 1C). Contrary to the

144 type 1 plaid, the type 2 stimulus plaid was composed of two component gratings of

145 different speeds, which resulted in the VA and IOC directions being different. The

146 speed of one component was twice as fast as the other $(35.6$ and $71.2 \mathrm{~mm} / \mathrm{s})$. The

147 spatial frequencies of the component gratings were $20.8 \mathrm{~mm} / \mathrm{cycle}$. The direction of

148 the slower component was either $\pm 18,54,90,126$ or $162^{\circ}$ from upward direction.

149 The direction of the faster component was always $30^{\circ}$ counterclockwise relative to

150 the slower component, resulting in the VA and IOC directions 20.1 and $66.2^{\circ}$

151 counterclockwise relative to the slower component, respectively.

\section{Procedure}

154 The task was to discriminate whether the direction of motion stimulus was orientated left- or rightward (Figure 2C). A trial started when the pigeon pecked a self-start key above the window illuminated with white LED. This light turned off and a motion stimulus appeared on the monitor. After 2 sec, two choice keys on the left and right of the window were illuminated by a red and blue LED. The pigeons were differentially reinforced by pecking the keys according to the direction of the 
160 stimulus. The left key was correct when the direction of motion stimulus tilted

161 leftward, and vice-versa. Food reward was delivered with a probability of 50\%.

162 Regardless of the delivery of the food, the light above the food hopper came on when

163 the subject's response was correct, serving as a secondary reinforcement. This

164 treatment was used to increase the number of trials per daily session. Pecking the

165 wrong key resulted in a 5-7 sec timeout and correction trials were repeated before

166 the next trial until the bird chose the correct key. In a correction trial, the same

167 stimulus appeared, and pecks to the wrong key were not counted in the 5th

168 correction trial. This treatment aimed to prevent response biases. After $3 \mathrm{sec}$ of

169 reinforcement or timeout, the self-start key was illuminated and the next trial (or

170 correction trial) started. The precise duration of access to food varied for each subject,

171 to keep its weight constant. The duration of timeout also varied for each subject:

172 shorter for pigeons who lost motivation and longer for pigeons whose performance

173 did not increase during training.

174 The pigeons were first trained with left- and rightward dot motion $\left( \pm 90^{\circ}\right)$ at $0 \mathrm{~cm}$

175 viewing distance. A daily session consisted of 200 trials $(2$ directions $\times 100$ repetitions). After accuracy of above $80 \%$ in one session, four other motion direction conditions were added ( \pm 54 and $126^{\circ}$, each left- and rightward $)$. Another four motion 
178 directions were added in the next phase $\left( \pm 18\right.$, and $162^{\circ}$, each left- and rightward).

179 After that, a type 1 plaid stimulus was presented on a half of session. The trial order

180 was randomized every session to prevent any order effect. The randomization was

181 conducted for every 20-trial block composed of two stimulus type (dot and plaid) and

18210 motion directions to prevent the same stimulus type or direction appearing on

183 successive trials. The block-based randomization was repeated 10 times for one

184 session consisting of 200 trials. The pigeons advanced to test sessions if their

185 performances on both dot and type 1 plaid conditions in one session was above $80 \%$.

186 A test session consisted of 180 training and 20 probe trials. All responses in probe

187 trials were reinforced, irrespective of the pigeon's choice. This was done to prevent

188 learning with probe stimuli using cues or criteria other than those in training trials.

189 In a probe trial, the type 2 plaid stimulus was presented. Probe trials were inserted

190 in a semi-randomized way so that they did not appear in two consecutive trials.

191 Between test sessions, at least one training session was conducted to check if

192 performance was above $75 \%$; if not, training sessions were repeated until

193 performance met the criterion.

194 After completing 10 test sessions in which performance on training trials was above

$19570 \%$, training sessions with dot and type 1 plaid stimuli were repeated with the 
monitor distance gradually increased (10 cm steps). If performance decreased to $70 \%$,

197 the monitor distance was manipulated using smaller steps $(3 \mathrm{~cm})$. Then the pigeons

198 advanced to test sessions with the $40 \mathrm{~cm}$ monitor distance and completed 10 sessions

199 in which training trial performance was above $70 \%$.

200

201 Analysis

202 A modified von Mises distribution, which is a circular distribution analogous to the

203 linear normal distribution, with four free parameters was fitted to the rate of right key

204 responses using least-square minimization with the Matlab function 'nlinfit.'

$$
\operatorname{logit}(R(\theta))=k_{1}+k_{2} \times \exp \left(k_{3} \times \cos \left(\theta-k_{4}\right)\right)
$$

The ratio of right key responses $R(\theta)$ was linearized with logit function. The parameter

$207 \quad k_{1}$ and $k_{2}$ define the upper and lower limits. The parameter $k_{3}$ and $k_{4}$ define the

deviance and peak position. The difference in peak positions between training and

probe data was calculated for each individual. To statistically test whether the pigeons' 


\section{Results}

216 All pigeons except for one (S4) completed 10 testing sessions in both viewing

217 conditions. Because S4's performance deteriorated during the test phase at $40 \mathrm{~cm}$, only

2188 test sessions at this distance were analyzed for this individual. The results are shown

219 in Figure 3. Figure 3A, C represent the proportion of right key choices and its fitted

220 curve as a function of direction of motion stimulus when the viewing distances were 0

221 and $40 \mathrm{~cm}$, respectively. The pigeons successfully discriminated the direction of dot

222 motion and type 1 plaid at both viewing distances (gray plots). The proportion of right

223 key choices increased as the motion was directed rightward. The response to type 2

224 plaid (black plots) was consistent with the VA rule for both viewing distances. The peak position of right key choice in probe trials shifted 19.7 and $25.6^{\circ}$ from baseline for the conditions of 0 and $40 \mathrm{~cm}$ viewing distances, respectively. The response to type 2 plaid was plotted as a function of direction of the slower component; hence these shifts were consistent with the VA direction $\left(20.1^{\circ}\right)$ rather than the IOC direction $\left(66.2^{\circ}\right)$. In Figure

229 3B, D the peak shift and its confidence interval were plotted for each bird at viewing 
232 the VA direction was significantly smaller than from the IOC direction $\left(X^{2}(1)=53.777\right.$,

$233 p=0.000, B_{s t d}=2.542$ ), and there was no effect of viewing distance (main effect, $X^{2}(1)=$

$2340.450, p=0.502, B_{\text {std }}=0.321$, interaction with the VA/IOC model, $X^{2}(1)=0.125, p=$

$2350.724, B_{s t d}=0.192$ ), indicating that for both viewing conditions the pigeons responded

236 based on the VA rule.

237

238 Discussion

239 In a study using a touch monitor procedure we previously found that pigeons

240 perceive different motion directions than humans from type 2 plaid stimuli.

241 Whereas humans integrate motion components in plaid based on the IOC rule,

242 pigeons perceive integrated motion based on the VA rule (Hataji et al. 2019). The

243 present study tested whether the species difference merely resulted from different

244 experimental settings because the viewing distance for pigeons was much closer

245 due to the use of touch panel. The present study demonstrated that pigeons

246 responded to plaid stimuli based on the VA rule at both 0 and $40 \mathrm{~cm}$ viewing

247 distances. Taken together with the earlier study using a different behavioral

248 procedure (pecking dots surrounding a motion stimulus), this result suggests that

249 pigeons and humans have different motion integration processes. 
250 The species difference in visual motion integration could be explained by the

251 difference in the dominant visual pathway and shape of receptive field. Pigeons are

252 known to depend on different visual pathways than primates (Shimizu and

253 Watanabe 2012). Primates depend on the thalamofugal pathway, which relays

254 visual signal from retina to the caudal part of telencephalon through a dorsal part

255 of thalamus, whereas pigeon retinal ganglion cells mainly project to the optic

256 tectum in midbrain. The receptive fields of motion sensitive neurons in optic

257 tectum have different shapes (Jassik-Gerschenfeld and Guichard 1972) compared

258 to visual Wulst, which is evolutionarily homologous to primary visual cortex of

259 mammals (Ng et al. 2010). It is not clear what phylogenetic and ecological factors

260 drive the evolution of the different visual pathways, but the present finding that

261 motion integration processes are different between the two species appears

262 relevant. For example, humans perceived VA direction when the plaid was

263 presented briefly, indicating that VA direction is computed faster than IOC

264 direction (Yo and Wilson 1992). This fast coding of motion information might be

265 crucial for fast flight in pigeons.

266 If the pigeons responded based on VA direction, it is possible that they simply responded probabilistically to both component directions. Another possible 
268 confound is that the pigeons responded to the $2 \mathrm{D}$ pattern of the plaid and not to

269 visual motion itself, specifically, because the VA direction is almost perpendicular

270 to the checkerboard orientation (see Figure 1C). If the pigeons responded to the

271 component direction or 2D pattern, their discrimination performance for type 1

272 plaid should decrease as the difference in component directions increases, because

273 the component directions deviate more from the VA direction and the checkerboard

274 shape changes from rectangle to square. Contrary to this hypothesis, the pigeons

275 performed better as difference in component directions increased (accuracy of $15-$

$27625^{\circ}, 68.4 \% ; 80-90^{\circ}, 81.7 \%, X^{2}(1)=80.482, p=0.000, B_{s t d}=0.420$, Supplementary

277 Figure S1), which rules out the possibility that the pigeons responded based on the

278 component direction or checkerboard shape.

279 Humans perceive the plaid motion in the VA direction when it is viewed in the

280 peripheral visual field (Yo and Wilson 1992). This indicates that different visual

motion processes work for the fine central and coarse peripheral visions. Pigeons

have two areas of retinal density in each eye: the lateral visual field projects onto

the central fovea and the frontal visual field projects onto the area dorsalis 
286 1971), and the velocity threshold and retinal projection are different for frontal and

287 lateral vision (Martinoya et al. 1983; Remy and Güntürkün 1991; Miceli et al.

288 2006). Therefore, different motion processes might work for the central and lateral

289 vision. In the present study, however, the pigeons looked at motion stimuli with

290 their frontal vision irrespective of viewing conditions. This may be because a $40 \mathrm{~cm}$

291 viewing distance is not enough to induce use of lateral vision, or the pigeons were

292 used to discriminating stimuli with frontal vision due to extensive training using

293 touch panel procedures. Thus, it remains unknown whether the species difference

294 in the perception of plaid motion reflects a difference in motion integration or the

295 visual field where the stimulus is projected. Further studies are needed to address

296 this issue by introducing a procedure to induce the use of lateral vision in pigeons.

298 Acknowledgment

299 This study was financially supported by JSPS KAKENHI Grant Numbers

300 15J02739 to YH and 16H01505 and 16H06301 to KF. We thank James R. Anderson

301 for editing the manuscript. 
303 Compliance with ethical standards

304 This study adhered to the ethical guidelines of Kyoto University, and was

305 approved by the Animal Experiments Committee of the Graduate School of Letters,

306 Kyoto University (No. 18-32).

307

308 Competing interests

309 The authors declare no conflicts of interest.

310

311 Data availability

312 The datasets generated or analyzed during the current study and the

313 supplementary stimulus movies and figures are available in the Open Science

314 Framework repository, https://doi.org/10.17605/OSF.IO/Z4UP3

315 


\section{References}

317 Adelson EH, Bergen JR (1985) Spatiotemporal energy models for the perception of motion. J Opt Soc Am A 2:284-299. https://doi.org/10.1364/JOSAA.2.000284

Adelson EH, Movshon JA (1982) Phenomenal coherence of moving visual patterns. Nature 300:523-525. https://doi.org/10.1038/300523a0

Binggeli RL, Paule WJ (1969) The pigeon retina: quantitative aspects of the optic nerve and ganglion cell layer. J Comp Neurol 137:1-18. https://doi.org/10.1002/cne.901370102

Blough PM (1971) The visual acuity of the pigeon for distant targets. J Exp Anal

Bowns L, Alais D (2006) Large shifts in perceived motion direction reveal multiple global motion solutions. Vision Res 46:1170-1177. https://doi.org/10.1016/j.visres.2005.08.029

Brainard DH (1997) The psychophysics toolbox. Spat Vis 10:433-436.

Goto K, Lea SE (2003) Discrimination of direction of movements in pigeons following previous experience of motion/static discrimination. J Exp Anal Behav 80:29-42. https://doi.org/10.1901/jeab.2003.80-29 
Hayes B, Holden AL (1983) The distribution of displaced ganglion cells in the retina of the pigeon. Exp Brain Res 49:181-188. https://doi.org/10.1007/BF00238578

Howard SR, Avargues-Weber A, Garcia JE, Stuart-Fox D, Dyer AG (2017) Perception of contextual size illusions by honeybees in restricted and https://doi.org/10.1098/rspb.2017.2278

Jassik-Gerschenfeld D, Guichard J (1972) Visual receptive fields of single cells in unrestricted viewing conditions. Proc R Soc B 284:20172278.

Martinoya C, Rivaud S, \& Bloch S (1983) Comparing frontal and lateral viewing in Res 8:375-385. https://doi.org/10.1016/0166-4328(83)90182-1 cells in the pigeon retina labeled via retrograde transneuronal transport of the 
352 Movshon JA, Newsome WT (1996) Visual response properties of striate cortical

353

354

355

356

357

358

359

360

361

362

363

364

365

366

367

368

369

neurons projecting to area MT in macaque monkeys. J Neurosci 16:7733-7741.

https://doi.org/10.1523/JNEUROSCI.16-23-07733.1996

Nakamura N, Watanabe S, Fujita K (2008) Pigeons perceive the EbbinghausTitchener circles as an assimilation illusion. J Exp Psychol Animal Behav Processes 34:375-387. http://dx.doi.org/10.1037/0097-7403.34.3.375

Nakamura N, Watanabe S, Fujita K (2014) A reversed Ebbinghaus-Titchener illusion in bantams (Gallus gallus domesticus). Anim Cog 17:471-481. https://doi.org/10.1007/s10071-013-0679-y

Ng BSW, Grabska-Barwińska A, Güntürkün O, Jancke D (2010) Dominant vertical orientation processing without clustered maps: early visual brain dynamics imaged with voltage-sensitive dye in the pigeon visual Wulst. J Neurosci 30:6713-6725. https://doi.org/10.1523/JNEUROSCI.4078-09.2010 (2010).

Qadri MA, Cook RG (2015) Experimental divergences in the visual cognition of birds and mammals. Comp Cogn Behav Rev 10:73-105. https://doi.org/10.3819/ccbr.2015.100004 (2015)

Remy M, Güntürkün O (1991) Retinal afferents to the tectum opticum and the nucleus opticus principalis thalami in the pigeon. J Comp Neurol 305:57-70. 

https://doi.org/10.1002/cne.903050107

371 Shimizu T, Watanabe S (2012) The avian visual system: overview. In: Lazareva

372 OFS, Shimizu T, Wasserman E (ed) How Animals See the World. Oxford University Press, Oxford, pp 473-482

374 Weintraub DJ (1979) Ebbinghaus illusion: context, contour, and age influence the judged size of a circle amidst circles. J Exp Psychol Hum Percept Perform 5:353-364. http://dx.doi.org/10.1037/0096-1523.5.2.353

Wylie DR, Gutiérrez-Ibáñez C, Gaede AH, Altshuler DL, Iwaniuk AN (2018) Visualcerebellar pathways and their roles in the control of avian flight. Front Neurosci 12:223. https://doi.org/10.3389/fnins.2018.00223 


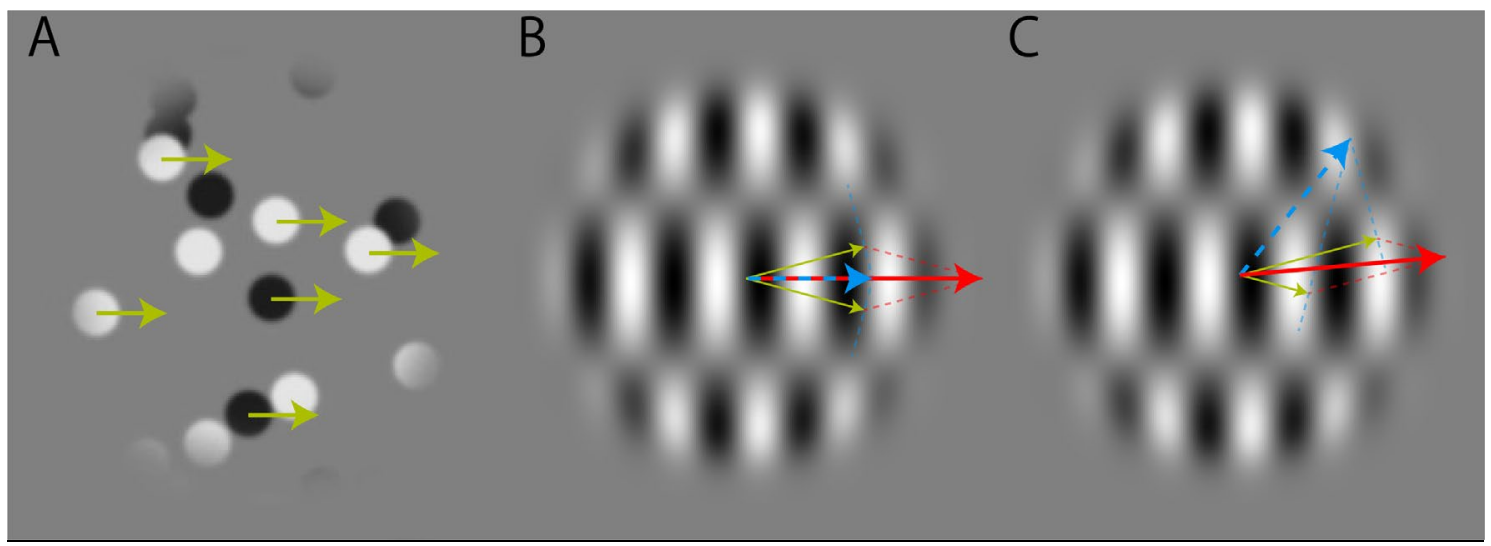

387 Figure 1 Stimuli used in the present study. (A) White and black dots assigned

388 randomly and moving in a specific direction with $100 \%$ coherence and (B) type 1

389 plaid motion composed of two drifting gratings of the same speed were used for

390 training stimuli. (C) Type 2 plaid motion, composed of gratings of different speeds,

391 corresponding to probe stimuli. Small yellow arrows represent vectors of dot and

392 component grating motion. Red and blue arrows represent the VA and IOC

393 directions, respectively. 
A

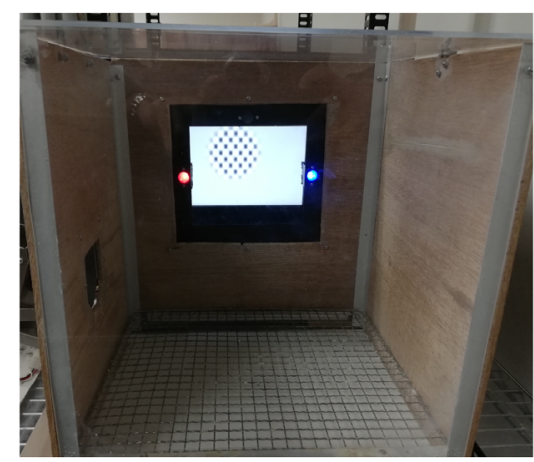

B

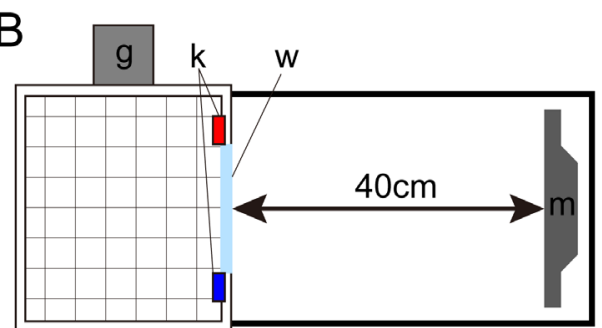

C

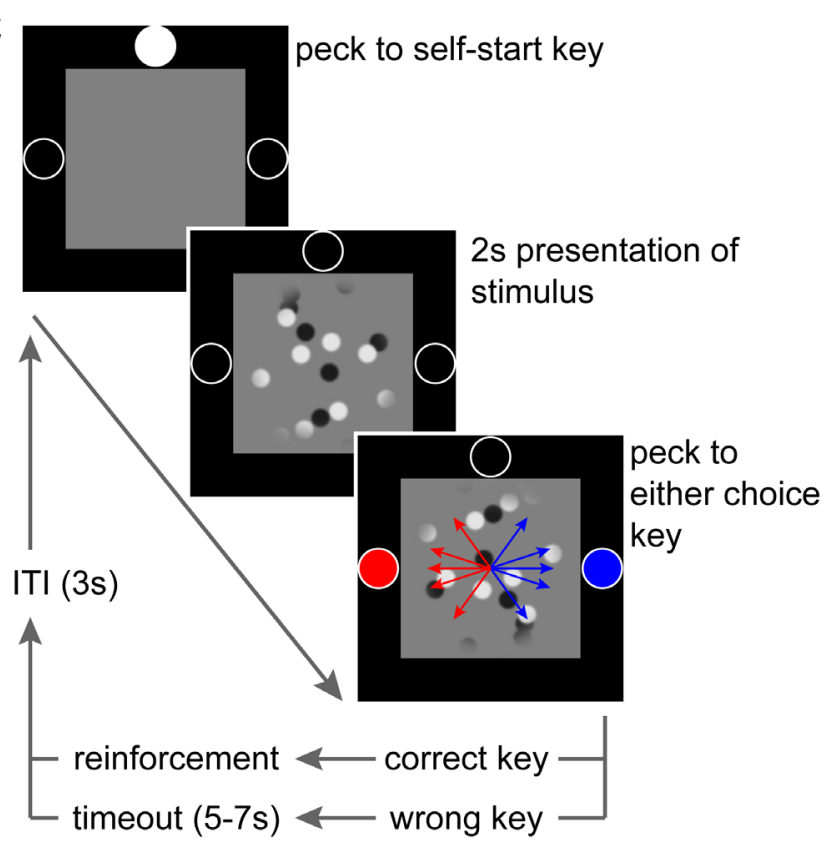

395

Figure 2 Apparatus and procedure. (A, B) The apparatus used in the present study.

A LCD monitor (m) was placed 0 or $40 \mathrm{~cm}$ from a transparent window (w) of an

operant chamber. Three response keys $(\mathrm{k})$ were attached on the front wall. A grain sequence of the motion discrimination task. The pigeons were trained to peck the rightward (represented with arrows on the stimulus). 


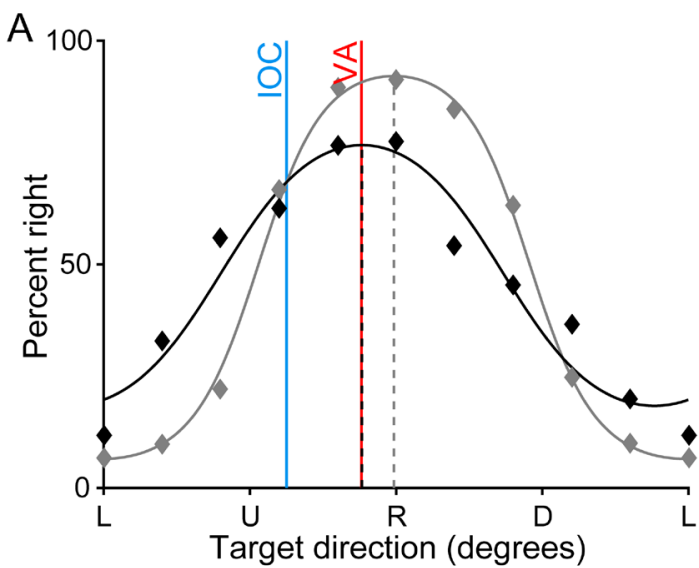

C

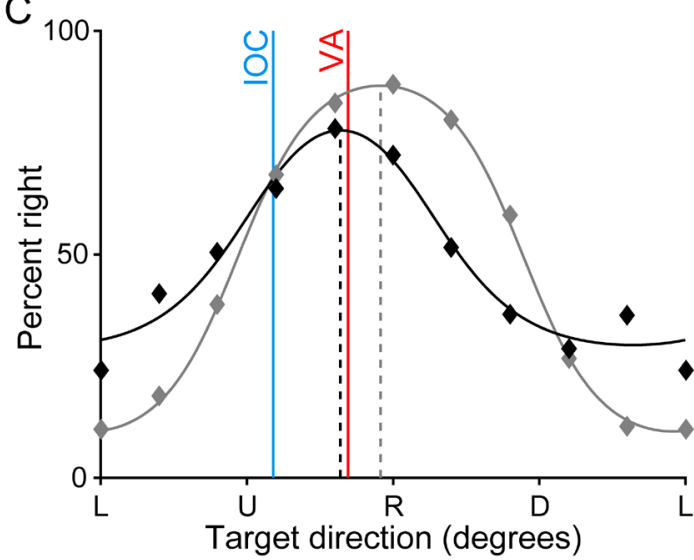

B
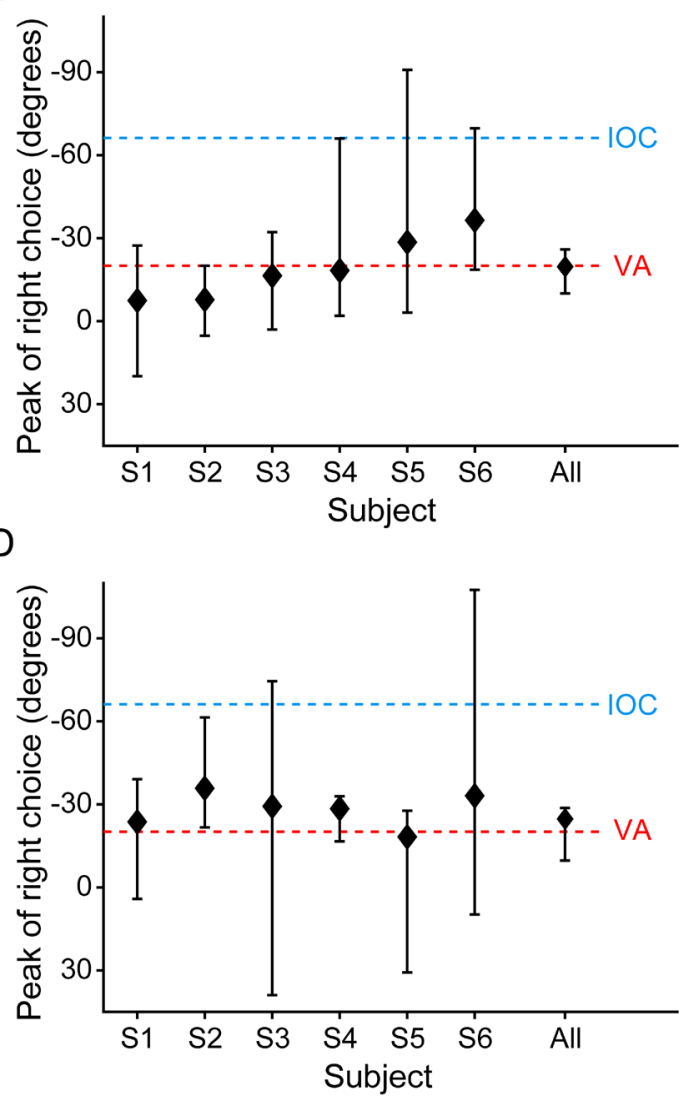

405 Figure 3 Proportion of right key choice and peak position of fitted function.

406 (A, C) The proportion of right key choices is plotted as a function of direction of

407 motion stimulus when the viewing distance was 0 (A) and $40 \mathrm{~cm}(\mathrm{C})$. For the

408 training data (gray plots), the horizontal axis represents the dot direction and

409 integrated direction of type 1 plaid (L: left, U: up, R: right, D: downward); for the

410 probe data (black plots), it represents the direction of slower component of type 2

411 plaid. Curved lines and vertical dotted lines represent the fitted function and its 
413 data should be placed based on the VA and IOC rules, respectively. (B, D) The shift

414 in peak position was calculated from the difference in peak positions between

415 training (gray dotted line in A, C) and probe conditions (black dotted line in A, C).

416 Error bars represent bootstrapped 95\% confidence intervals. Red and blue dotted

417 lines represent the VA and IOC directions, respectively.

418 